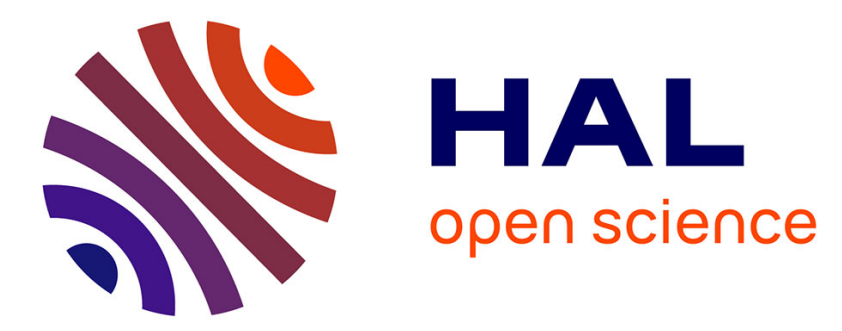

\title{
Handling of radical prostatectomy specimens: Total or partial embedding?
}

Ben Vainer, Birgitte Grønkær Toft, Karen Ege Olsen, Grete Krag Jacobsen, Niels Marcussen

\section{- To cite this version:}

Ben Vainer, Birgitte Grønkær Toft, Karen Ege Olsen, Grete Krag Jacobsen, Niels Marcussen. Handling of radical prostatectomy specimens: Total or partial embedding?. Histopathology, 2011, 58 (2), pp.211. 10.1111/j.1365-2559.2011.03741.x . hal-00614753

\section{HAL Id: hal-00614753 https://hal.science/hal-00614753}

Submitted on 16 Aug 2011

HAL is a multi-disciplinary open access archive for the deposit and dissemination of scientific research documents, whether they are published or not. The documents may come from teaching and research institutions in France or abroad, or from public or private research centers.
L'archive ouverte pluridisciplinaire HAL, est destinée au dépôt et à la diffusion de documents scientifiques de niveau recherche, publiés ou non, émanant des établissements d'enseignement et de recherche français ou étrangers, des laboratoires publics ou privés. 


\section{Histopathology}

\section{Handling of radical prostatectomy specimens: Total or partial embedding?}

\begin{tabular}{|c|c|}
\hline Journal: & Histopathology \\
\hline Manuscript ID: & HISTOP-11-09-0645.R4 \\
\hline Manuscript Type: & Original Article \\
\hline $\begin{array}{r}\text { Date Submitted by the } \\
\text { Author: }\end{array}$ & 08-Mar-2010 \\
\hline Complete List of Authors: & $\begin{array}{l}\text { Vainer, Ben; Rigshospitalet, University of Copenhagen, Dept. of } \\
\text { Pathology } \\
\text { Grønkær Toft, Birgitte; Rigshospitalet, Dept. of Pathology } \\
\text { Ege Olsen, Karen; Odense University Hospital, Dept. of Pathology } \\
\text { Krag Jacobsen, Grete; Rigshospitalet, Dept. of Pathology } \\
\text { Marcussen, Niels; Odense University Hospital, Dept. of Pathology }\end{array}$ \\
\hline Keywords: & prostate cancer, radical prostatectomy \\
\hline
\end{tabular}

\section{S ScholarONE" \\ Manuscript Central}




\title{
Handling of radical prostatectomy specimens: Total or partial embedding?
}

\author{
Ben Vainer ${ }^{1}$, Birgitte Grønkær Toft ${ }^{1}$, Karen Ege Olsen ${ }^{2}$, Grete Krag \\ Jacobsen ${ }^{1}$, Niels Marcussen ${ }^{2}$ \\ ${ }^{1}$ Department of Pathology, Rigshospitalet, University of Copenhagen, Denmark \\ ${ }^{2}$ Department of Pathology, Odense University Hospital, Denmark
}

Short running title: Handling of RPS

Keywords: Gleason; pathology; partial embedding; prostate cancer; radical prostatectomy.

Correspondence:

Ben Vainer, MD, PhD, Dept. of Pathology, Rigshospitalet, Blegdamsvej 9, DK-2100

Copenhagen, Denmark. Tel.: +45 35455396; Fax: +45 35455414; e-mail:

ben.vainer@rh.regionh.dk 


\begin{abstract}
Proper examination and accurate reporting of radical prostatectomy specimens (RPS) is essential in determining post-surgical treatment and predicting patient outcome. Surveys have demonstrated the absence of consensus on handling of RPS. Aims: To determine whether significant information is lost when only half of the horizontal tissue sections are examined. Methods and Results: During a one-year period, 238 RPS were sectioned into horizontal slices. Apex and basis was cut sagitally, and remaining slices were embedded in quadrants. Glass slides from every second horizontal slice were withheld. The remaining slides were evaluated microscopically, and essential pathological parameters were recorded. Subsequently, a full report was compiled, including the withheld slides. A median of 12 slides (30\%) were withheld during initial assessment. In 8 RPS (3.2\%) the pTNM stage had to be changed; in 6 cases (2.6\%) from pT2b to pT2c and in 2 cases $(0.8 \%)$ from pT2c to pT3a. In 1 RPS $(0.4 \%)$ the surgical margin status was changed.
\end{abstract} Conclusions: Only little information is lost with systematic partial embedding, overlooking features significant for the postoperative treatment in only 1.2\%. Partial embedding as suggested, decreasing the laboratory workload by $30 \%$, is concluded to be acceptable for valid histopathological assessment. 


\section{Introduction}

Proper examination and accurate reporting of radical prostatectomy specimens (RPS) is crucial in decision of post-surgical treatment and prediction of patient outcome. Important histological features are tumour grade, tumour volume, tumour stage and surgical margins. There is widely international agreement that tumour grade should be assessed according to the Gleason criteria, which in 2005 was modified for better correlation with prognosis by an international working group under International Society of Urological Pathology (ISUP) 1,2 .

Macroscopical recognition of cancer areas within the prostatic gland is inaccurate, and macroscopical naked eye guidance for selection of sections for embedding may therefore not provide the correct areas for histopathological evaluation. Consequently, the entire RPS is sectioned and prepared for microscopical evaluation. Mostly, the gland is cut in serial horizontal sections, and the apex and the bladder neck area are cut parasagitally. Consensus of the proper way to handle RPS has, however, not yet been obtained, clearly illustrated by a survey of American pathologists from 1994 and a recent European survey, conducted by the European Network of Uropathologists (ENUP) ${ }^{3,4}$.

With the increasing demands to surgical pathology, the continuous difficulties in recruiting staff, and the limitations of economical resources it is inevitable to set priorities in the pathology service. Whether total embedding of RPS is in fact needed in order to provide the urologists with the correct information, or whether this information is sufficiently obtained with examination of only selected parts of the prostate has not been approached systematically. Thus, the aim of the present study was to evaluate whether essential information is lost when less than the whole prostate gland is embedded for histopathological examination. 


\section{Materials and methods}

A total of 238 RPS were included constituting all prostatectomies performed at two Danish university hospitals (i.e. Rigshospitalet/Copenhagen University Hospital, and Odense University Hospital) during a 12 months period from September 2007 to August 2008.

\section{Handling of the surgical specimen}

When arriving at the pathology department, the prostate was partly divided by a cut in the mid-sagital plane through the anterior surface, separating the two lobes for optimal fixation. The gland was fixed for an additional $20 \mathrm{hrs}$ in formic acid and $24 \mathrm{hrs}$ in $4 \%$ buffered formalin. The procedure at Odense University Hospital was slightly different, as the initial mid-sagital cut was not performed, and decalcification was done after sectioning. The gross examination included measurement in three dimensions, weighing the prostate after removal of the seminal vesicles, and separating the left from the right lobe after inking the anterior and the posterior halves with two different colours. Apical and basic sections of 5-10 mm depending of the total size of the RPS were cut horizontally, subsequently sliced parasagitally and placed in cassettes with often more than one section per cassette. The remaining part of the prostate was cut horizontally in appr. $3 \mathrm{~mm}$ sections and placed in standard cassettes, ensuring laterality. Large sections were divided to fit standard cassettes. Finally, sections from the seminal vesicles (as a minimum the apex and a cross section) were embedded.

Post-fixation in $4 \%$ formalin and embedding in paraffin was followed by $4 \mu \mathrm{m}$ sectioning and staining with haematoxylin and eosin.

\section{Microscopical evaluation}

The pathologist divided the glass slides from each specimen in two sets by removing the haematoxylin and eosin stained sections representing every second horizontal slice of the middle part of the prostate (Fig. 1). These glass slides were kept separately. In some cases, an uneven number of slides were withheld due to organ assymmetry and/or variation in slice thickness of the two lobes. The remaining glass slides, including those from the apex, the basis and the seminal vesicles, were evaluated by conventional light 
microscopy, if necessary with additional immunohistochemical staining with a cocktail of racemase P504S, high molecular weight-cytokeratin, and p63. A form was filled out for each prostate containing information of tumour type (WHO 2004 criteria $^{5}$ ), Gleason score with elaboration of primary and secondary growth pattern according to the 2005 WHO/ISUP consensus report ${ }^{1}$ with the clarification of how to deal with tertiary growth patterns (recently reviewed by Epstein ${ }^{6}$ ), rough estimation of the volume involved (\%), diameter of the largest malignant focus $(\mathrm{mm})$, laterality, multifocality, extracapsular extension, involvement of the resection margin, and tumour stage (pTNM system ${ }^{5}$ ). Tertiary Gleason patterns were dealt with according to the precision recently described in a review Epstein. The extent of extraprostatic growth (stage pT3) was recorded as $\leq 2$ or $>2$ high power fields. After completion of this assessment, the initially withheld slides were examined, and a final report was prepared for the urologists.

\section{Statistics}

Medians or means are presented depending on data distribution. Student's t-test was applied for group comparisons, and the Spearman test or the Kruskal Wallis test was used for assessment of trends. A $p$ value of 0.05 or less was considered statistical significant.

The study was conducted according to the Helsinki Declaration and the Danish legislation.

\section{Results}

Details of the 238 radical prostatectomies are shown in Table 1. The age of the patients was 63.4 years (mean; SD 5.2, range 47-84) with no difference between the two centres. The prostate glands with a median weight of $41.1 \mathrm{~g}$ (range 16-126 g) were embedded in median 38 cassettes (range 18-76), and between 3 and 26 slides (median 12) were initially withheld. This corresponded to $29.7 \%$ of the total slides (range 14.3-55.8\%). The number of cassettes as well as the number of initially removed slides correlated with the prostatic weight (total no. of cassettes, $R_{S}=0.872$; no. of initially removed slides, $R_{S}=0.776$; both $\mathrm{p}<0.001$, Spearman's test) (Fig. 1). 
Adenocarcinoma was demonstrated in all 238 RPS, of which 235 (98.7\%) were classified as acinar. Alternative growth features were demonstrated in 29 acinar adenocarcinomas ${ }^{1}$ $(12.3 \%)$ (Table 1$)$. The remaining 3 cases $(1.2 \%)$ were either pure ductal $(n=1)$ or mixed acinar and ductal $(n=2)$ adenocarcinomas. Twenty-one (8.8\%) of the carcinomas involved only one lobe, all of which involved less than half of the lobe corresponding to stage pT2a. No stage pT2b tumours were registered. One-hundred-fifty $(63.0 \%)$ were bilateral but confined to the prostate gland (pT2c), whereas extraprostatic growth, i.e. stage pT3, was observed in 67 (28.2\%) with $48(20.2 \%)$ having spread to the extraprostatic connective tissue only (pT3a) and 19 (8.0\%) involving the seminal vesicles (pT3b). No stage pT4 tumours were diagnosed. Statistical testing revealed an inverse correlation between the tumour stage and the prostatic weight, i.e. high-stage carcinomas tended to be present in smaller prostate glands $\left(R_{S}=-0.137, p=0.035\right)$. Distribution of the tumour volume was strongly skewed to the right with a median of $10 \%$ (range $1-80 \%$ ) and a median diameter of the largest cancer focus of $15.0 \mathrm{~mm}$.

The dominant Gleason score was 7 ( $n=173,72.7 \%)$, including an upgrading from diagnostic biopsies in 58 cases $(p<0.001)$. When examining the withheld slides, the Gleason score had to be changed in five cases, namely in one case from $3+4=7$ to $3+5=8$, in three cases from $4+3=7$ to $4+4=8$ and in 1 case from $3+4=7$ to $4+5=9$. Two cases were downgraded from $3+4=7$ to $3+3=6$ with a tertiary pattern 4 component. In the group of Gleason score 7 carcinomas, distribution of primary and secondary growth patterns changed in three cases, as the amount of the secondary pattern was dominating in the initially withheld sections (in two cases from $3+4$ to $4+3$ and in one case from $4+3$ to $3+4$ ).

In two cases $(0.8 \%)$, small foci of extraprostatic growth were only detected in the withheld sections but not in the originally examined slides, which changed the stage from pT2c to pT3a, and in one RPS (0.4\%) a small focus of adenocarcinoma was detected in the surgical margin only in the initially withheld slides. In six other cases, the stage was changed from pT2a to pT2c because of revealing of involvement of both lobes. No significant changes were found in tumour volume or in diameter of the largest tumour focus. 


\section{Discussion}

Correct histopathological evaluation of RPS, including tumour grading and staging, is essential for the choice of the successive treatment and of prediction of prognosis ${ }^{7}$. to obtain this, correct tissue sampling from the specimens is of utmost importance. Ideally, all tissue should be examined under the microscope, but this is not always feasible due to shortage of staff, time and economy. Therefore, selection of tissue for microscopical analysis must be performed. Usually, pathologists select tissue sections of interest for microscopical assessment based on different, more or less reliable, macroscopical criteria. Mostly, the gross appearance is an acceptable guidance, but in some cases this is not the case. Some neoplasias, such as prostate cancer, are macroscopically not easily recognisable within the specimen, and selection might therefore be more or less at random and, hence, seriously flawed.

The sampling technique for RPS and subsequent microscopical evaluation differs between centres, and consensus for the handling of RPS has not yet been reached. A recent survey among European uropathologists published in 2008 demonstrated that $71.6 \%$ of the institutions always embedded RPS in total, additional $17.6 \%$ performed total embedding in selected cases, and more than $10 \%$ of the institutions handled RPS always by partial embedding ${ }^{4}$. The survey did not describe how the selection was done when embedding partially, i.e. systematically or guided by gross appearance or other specified criteria.

Several studies have been conducted in which embedding of whole mount sections have been compared to embedding of quadrants, the conclusion being that whole mount sampling is not superior to evaluation on standard-sized glass slides as to detection of adverse pathological features ${ }^{8,9}$. Also, as Srigley pointed out, whole-organ sectioning is expensive and requires additional technical expertise in the laboratory and important prognostic information may be lost primarily due to the thickness of the sections ${ }^{10}$.

The present study demonstrated that partial embedding of RPS with systematical selection of every second whole coronal section of the major part of the prostate and total embedding of the apical and basal sections loses only minor information important for the planning of post-surgical treatment and follow-up. This was corroborated by Kim et al ${ }^{11}$, 
who analyzed 148 RPS in order to evaluate the impact of five partial sampling methods on tumour stage, Gleason score, extraprostatic extension, resection margin, and number of cassettes. Alternative slices plus peripheral 3-mm rim of the remaining prostate was most efficient. This method detected all cases of extraprostatic extension and resection margin involvement and reduced the number of paraffin blocks by $25 \%$.

For cases where cancer is not easily identified in the primarily examined histological slides, the remaining tissue should be examined and if malignancy is still not found cutting deeper should be done into the blocks corresponding to the location of malignancy demonstrated in the diagnostic needle biopsies. In the one case in the present investigation in which cancer was not detected in the initial slides, the remaining prostatic tissue would under any circumstance have been embedded for histological assessment. Information for guiding the post-surgical approach and for prognosis was therefore lost in only three cases $(1.2 \%)$ when embedding only half of the horizontal sections from the RPS, including two cases that changed tumour stage from organ-confined cancer (pT2) to cancer with minimal foci of extraprostatic extension (pT3) and one case in which positive surgical margins were demonstrated. In routine urology, such changes do not alone prompt a shift in the post-surgical approach.

The procedure for safe-keeping the initially unembedded prostate slices should be considered, depending on how detailed information, the urologists need in cases of minimal cancers not detected at first. Consecutive embedding of all slices in order and only processing half of the horizontal slices for microscopic examination will ensure a precise localisation of minimal cancer foci, but is obviously not as effective in decreasing the laboratory workload. We have concluded that separation of the right and the left lobe in separate containers is sufficient in these cases.

In some clinical trials, the inclusion criteria comprise separation of Gleason score $7(3+4)$ from $7(4+3)$. In the present study, advancement from 3+4 to $4+3$ was observed in only two cases ( $0.8 \%$ of the total number of RPS; $1.2 \%$ of Gleason score 7 cancers), whereas one case was downgraded from $4+3$ to $3+4$. However, inclusion into such trials is always combined with other criteria, e.g. pT stage and surgical margin status often with inclusion of pT2c tumours with positive resection margins. This was the case of one of the two cases that changed from $3+4$ to $4+3$ when assessing the entire gland $(0.4 \%)$. The 
prognostic significance of $7(3+4)$ vs. $7(4+3)$ is not adequately documented, and the exclusion of such patients from participation in those clinical trials by the systematic partial embedding protocol applied in this study may therefore not influence the chance of survival for this patient.

Recognising the increasing demands to surgical pathology concerning both the increasing number of specimens and their complexity combined with the continuous difficulties in recruiting staff, it is important to decrease the workload whenever possible without compromising with the information needed to treat the patients optimally. In this respect, a reduction of tissue sections is a central issue. The approach with partial embedding of tissue from RPS used in this investigation will decrease the number of cassettes by approximately $30 \%$, which in a cancer centre such as Rigshospitalet with more than 250 prostatectomies annually (increase of 50\% from 2008 to 2009) will result in a fall in workload at the pathology laboratory equivalent to 75 RPS annually.

In conclusion, systematic partial embedding of RPS loses only minor information, which for the great majority of patients does not influence their post-surgical treatment or follow-up, but results in a significant reduction of the workload and of the resource requirement. 


\section{References}

1. Epstein JI, Allsbrook WC, Amin MB, et al. The 2005 International Society of Urological Pathology (ISUP) consensus conference on Gleason grading of prostatic carcinoma. Am J Surg Pathol. 2005;29:1228-42.

2. Epstein JI, Allsbrook WC, Amin MB, et al. Update on the Gleason grading system for prostate cancer. Adv Anat Pathol. 2006;13:57-9.

3. True LD. Surgical pathology examination of the prostate gland. Practice survey by American society of Clinical Pathologists. Am J Clin Pathol. 1994;102:572-9.

4. Egevad L, Algaba F, Berney DM, et al. Handling and reporting of radical prostatectomy specimens in Europe: a web-based survey by the European Network of Uropathology (ENUP). Histopathology. 2008;53:333-9.

5. Epstein JI, Algaba F, Allsbrook WC, et al. Tumours of the prostate. In: Eble JN, Sauter G, Epstein JI, et al, eds. Tumours of the urinary system and male genital organs. Lyon: IARC Press; 2004:160-215.

6. Epstein Jl. An update on the Gleason grading system. J Urol. 2010;183:433-40.

7. Bostwick DG, Montironi R. Evaluating radical prostatectomy specimens: therapeutic and prognostic importance. Virchows Arch. 1997;430:1-16. 
8. Hollenbeck BK, Bassily N, Wei JT, et al. Whole mounted radical prostatectomy specimens do not increase detection of adverse pathological features. J Urol. 2000;164:1583-6.

9. Cohen MB, Soloway MS, Murphy WM. Sampling of radical prostatectomy specimens. How much is adequate? Am J Clin Pathol. 1994;101:250-2.

10. Srigley $\mathrm{J}$. Key issues in handling and reporting radical prostatectomy specimens. Arch Pathol Lab Med. 2006;130:303-17.

11. Kim K, Pak PJ, Ro JY, et al. Limited sampling of radical prostatectomy specimens with excellent preservation of prognostic parameters of prostate cancer. Arch Pathol Lab Med. 2009;133:1278-84. 


\section{Figure legends}

Figure 1. Gross examination and sectioning of a small radical prostatectomy specimen. After weighing and colour marking, the prostate was divided into the right and the left lobe by a mid-sagital cut, and the seminal vesicles were removed. Each lobe was sectioned separately by coronal slicing and further parasagital sectioning of the apical and basal horizontal slices. From the seminal vesicle the apex and a middle cross section was obtained. The photograph shows sectioning of the right lobe with a rest of the right seminal vesicle in the lower left corner and the entire left lobe with the seminal vesicle in the lower right corner. Sections withheld in the initial assessment are marked by red crosses. Because of the small size of the prostate, the hemiprostate sections were not further divided into quadrants in this particular case. 
Table 1. Characteristics of the radical prostactomies and corresponding pre-operative histopathology information. Unless otherwise stated, data are from evaluation of the radical prostatectomy specimens.

\begin{tabular}{|c|c|c|c|}
\hline No. of radical prostactomies & $\mathrm{n}=$ & 238 & (RH: 172, OUH: 66) \\
\hline Age of patients, years & (mean) & 63.4 & (range 47-84) \\
\hline \multicolumn{4}{|l|}{ Gleason, pre-op. biopsies ${ }^{(1)}$} \\
\hline 5 & (n) & 1 & $(0.4 \%)$ \\
\hline 6 & (n) & 100 & $(42.0 \%)$ \\
\hline 7 & (n) & 115 & $(48.3 \%)$ \\
\hline 8 & (n) & 10 & $(4.2 \%)$ \\
\hline 9 & (n) & 3 & $(1.3 \%)$ \\
\hline Missing & (n) & 9 & $(3.8 \%)$ \\
\hline Prostatic weight & (g; median) & 47.1 & (range 16-126) \\
\hline No. of cassettes/total slides & (median) & 38 & (range 18-76) \\
\hline \multirow[t]{2}{*}{ No. of slides initially removed } & (median) & 12 & (range 3-26) \\
\hline & (fraction - \%) & 29.9 & (range 14.3-55.8) \\
\hline \multicolumn{4}{|l|}{ Type of carcinoma } \\
\hline Acinar adenocarcinoma & (n) & 235 & $(98.7 \%)$ \\
\hline Conventional, no variants & (n) & 206 & (86.6 \% of all carcinomas) \\
\hline Glomeruloid variant & (n) & 4 & (1.7\% of all carcinomas) \\
\hline Mucinous extravasation & (n) & 8 & (3.4\% of all carcinomas) \\
\hline Foamy cell variant & (n) & 9 & (3.8\% of all carcinomas) \\
\hline Colloid variant & (n) & 6 & (2.5\% of all carcinomas) \\
\hline Pseudohyperplastic variant & (n) & 2 & (0.8\% of all carcinomas) \\
\hline Ductal adenocarcinoma & (n) & 1 & $(0.4 \%)$ \\
\hline Mixed acinar and ductal & (n) & 2 & $(0.8 \%)$ \\
\hline \multicolumn{4}{|l|}{ Gleason ${ }^{(1)}$} \\
\hline 5 & (n) & 7 & $(2.9 \%)$ \\
\hline 6 & (n) & 44 & $(18.5 \%)$ \\
\hline 7 & (n) & 173 & $(72.7 \%)$ \\
\hline $3+4$ & (n) & 112 & (64.7\% of Gleason $7 s)$ \\
\hline $4+3$ & $(n)$ & 61 & (35.3\% of Gleason $7 s$ ) \\
\hline 8 & (n) & 11 & $(4.6 \%)$ \\
\hline 9 & (n) & 6 & $(2.5 \%)$ \\
\hline Perineural growth & (positive; $\mathrm{n}$ ) & 185 & $(77.7 \%)$ \\
\hline Tumour diameter & (mm; median) & 15 & (range 1-45) \\
\hline Tumour fraction of total gland (volume) & (\%; median) & 10 & (range 1-80) \\
\hline \multicolumn{4}{|l|}{ Stage $(\mathrm{pT})^{(2)}$} \\
\hline pT2 & (n) & 171 & $(71.8 \%)$ \\
\hline$p T 2 a$ & $(n)$ & 21 & $(8.8 \%)$ \\
\hline$p T 2 b$ & (n) & 0 & $(0.0 \%)$ \\
\hline$P T 2 c$ & (n) & 150 & $(63.0 \%)$ \\
\hline pT3 & & 67 & $(28.3 \%)$ \\
\hline$p T 3 a$ & $(n)$ & 48 & $(20.2 \%)$ \\
\hline$p T 3 b$ & $(n)$ & 19 & $(8.0 \%)$ \\
\hline pT4 & (n) & 0 & $(0.0 \%)$ \\
\hline Involvement of surgical margins & (positive; $\mathrm{n}$ ) & 68 & $(28.6 \%)$ \\
\hline
\end{tabular}

${ }^{1}$ Gleason grading according to the ISUP 2005 modified scoring system (Ref. 1).

2 Tumour staging according to the WHO 2004 staging system (Ref. 7).

$\mathrm{SD}=$ standard deviation (normal distribution assumed) 


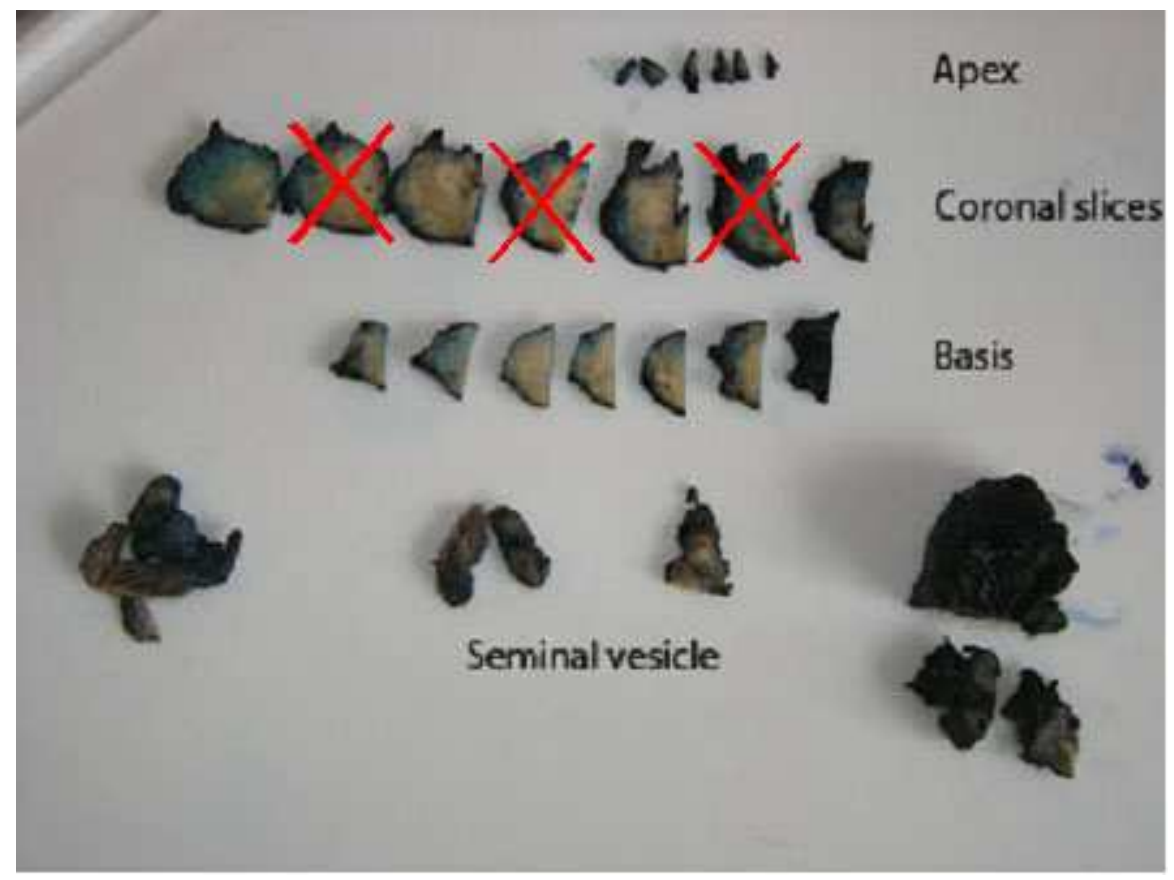

Figure 1. Gross examination and sectioning of a small radical prostatectomy specimen. After weighing and colour marking, the prostate was divided into the right and the left lobe by a midsagital cut, and the seminal vesicles were removed. Each lobe was sectioned separately by coronal slicing and further parasagital sectioning of the apical and basal horizontal slices. From the seminal vesicle the apex and a middle cross section was obtained. The photograph shows sectioning of the right lobe with a rest of the right seminal vesicle in the lower left corner and the entire left lobe with the seminal vesicle in the lower right corner. Sections withheld in the initial assessment are marked by red crosses. Because of the small size of the prostate, the hemiprostate sections were not further divided into quadrants in this particular case. $155 \times 117 \mathrm{~mm}(72 \times 72 \mathrm{DPI})$ 